\section{Digital Scanning, Archiving, and Transmitting Electron Micrographs}

\author{
Joiner Cartwright, Jr. \\ Baylor College of Medicine \\ joiner@bcm.tmc.edu
}

The primary responsibility of the clinical electron microscopy laboratory is to produce, and deliver to the pathologist or clinician, high quality ultrastructural images of pathological specimens in a timely manner. In order to facilitate this process, medical EM labs are converting from traditional chemical processing of photographic film and paper to the acquisition and transmission of electron images by computer. Ideally the electron microscope would incorporate a digital camera in the column to collect the images, thus bypassing chemical processing of film and paper altogether. However, due mainly to the high cost of the in-column cameras, many labs continue to use film, but replace the time consuming enlarging/printing in the darkroom with digital techniques at the computer. Even though film is still used, substantial savings in time, cost and filing space for prints are realized by digitizing the images from the negatives. Another important advantage to digital imaging is that photographic prints do not have to be retrieved for archiving and quality assurance purposes. The images never leave the lab.

This article describes the procedure we use to digitize electron images from Kodak Electron Microscope Film 4489 negatives using a MicroTek ScanMaker 8700 flat bed scanner using LaserSoft SilverFast software. Also described is our procedure for processing and archiving the images using a Windows XP based computer and Adobe PhotoShop image processing software. Images are transmitted to the requesting physician by file transfer protocol (FTP) via the internet and archived on CD/ROM.

\section{Scanning E.M. Negatives}

The MicroTek ScanMaker 8700 is a "dual bed" scanner. That is, it accepts "reflective" or opaque items on the top bed, face down on the glass under the top lid. It also accepts transparencies, either positive or negative, in the lower bed. 4" X 5" films, $6 \mathrm{~cm}$ films, mounted $35 \mathrm{~mm}$ transparencies, or $35 \mathrm{~mm}$ film strips with a maximum of six frames, are held in glassless "templates" specifically designed for that film size and laid into the "Main Film Holder" which is then slid into the lower bed slot. For other film sizes, including the 3.1/4" X 4" Kodak Electron Microscopy film (No. 4489), the films are laid onto the glass of the "Glass Film Holder" and inserted into the lower bed. In any case the item to be scanned is placed viewedsurface down with the top toward the front of the scanner. Notched sheet film negatives are placed onto the glass film holder with the notch to the left and toward the left rear corner of the scanner. Six Kodak No. 4489 negatives can be laid out on the glass film holder at one time. Lay them out as squarely as possible in the center of the glass, equidistant from the sides and ends of the glass. Insert the glass film holder into the lower bed slot gently and smoothly so that the films do not slide around.

Following is our procedure for scanning images from the negatives using LaserSoft's SilverFast scanning software, and then processing, transmitting and archiving the scanned images. The settings described are for scanning 31/4" X 4" Kodak Electron Microscopy film, No. 4489, in the Windows environment. However, the SilverFast distribution disk comes with both Windows and
Macintosh versions. SilverFast comes with basic image editing functions; however an image editor such as PhotoShop is still needed for E.M. images. SilverFast can be installed to open from within PhotoShop (File | Import | SilverFast) or to operate independently from PhotoShop. It is easier to access SilverFast from PhotoShop when you are scanning from negatives requiring differing settings. However it is even easier, when working with a homogeneous series of negatives where the scanned areas are the same size, to scan in all of the images and then process the scanned images in a separate session.

Turn the scanner on and open the LaserSoft SilverFast application on the computer. You may see previously scanned images in the right hand pane. Ignore them. In the left hand pane, establish the following settings:

\section{General Tab:}

$\begin{array}{lll}\text { Scan Mode } & \rightarrow & \text { Normal (file) } \\ \text { Original } & \rightarrow & \text { Transparency } \\ \text { Pos/Neg } & \rightarrow & \text { Negative } \\ \text { Frame-Set } & \rightarrow & \text { Save } \\ \text { Frame Tab: } & & \\ \text { Scan Type } & \rightarrow & 14 \rightarrow 8 \text { Bit Grayscale } \\ \text { Filter } & \rightarrow & \text { None } \\ \text { Setting } & \rightarrow & \text { Save } \\ \text { Image Type } & \rightarrow & \text { Standard } \\ \text { Name } & \rightarrow & \text { Name or number you assign to first image } \\ \text { (Size of) Original: } & 3.9 \text { in. horiz., scale } 100 \% \\ & 3.0 \text { in. vert., scale } 100 \% \\ \text { (Size of) Output: } & 3.9 \text { in. horiz.; } 3.0 \text { in. vert. } \\ \text { Q-factor Screen: } & 1.5333 \text { lpi M byte: } 2.79 \\ \text { Resolution (slider): } 500 \text { dpi } \\ \text { Negative Window: } \\ \text { (film brand): } & \rightarrow \text { Kodak } \\ \text { (film type): } & \rightarrow & <0 \text { other }> \\ \text { ASA: } \quad \rightarrow & \text { B\&W } 400\end{array}$

Brightness slider: Set initially to 0.0. Adjust as necessary. Auto tolerance: mid scale

NOTE: The settings here are chosen to give a good balance between file size, ease and speed of handling, and image quality. If you prefer to invert the image from negative to positive in PhotoShop rather than letting the scanner do it, specify Pos/Neg $\rightarrow$ Positive in the General tab above. You will not see the Negative Window then.

\section{Click Prescan}

The Prescan is a "quick \& dirty" scan that takes in the entire area of the film holder to locate the images or parts of an image that you want to scan. Be patient. It takes a while to begin scanning after clicking $\underline{\text { Prescan }}$. Positive images of the film negatives will appear along with a dotted "marquee" scan frame outlining the area to be scanned at high resolution. Although the scan frame will always be rectangular, and its size is determined initially by the size settings under the Frame Tab above, it can be altered by clicking and dragging the corners or sides. The size settings given here reflect the size of the actual image area on the film coming out of our scope. If you do change it by clicking and dragging, it will change the size settings you specified previously under the Frame Tab. The scan frame can 
now be dragged to any position on the prescanned area. Left click and hold anywhere inside the scan frame and drag it into position over the first film. The sides of the scan frame should coincide with the corresponding edges of the image area on the film. One side of the scan frame can be moved outward a little to include any printed negative numbers, mag values, etc. on the film. This will also give room outside of the image area to add a printed identifier to the image from within PhotoShop. If each of the negatives have the same image area, once the scan frame is adjusted to the first image, its size and shape need not be changed. It's necessary only to move the frame to subsequent images.

Open the frame tab in the left pane and enter a file name for the first image.

Click on Image Auto-adjust button, the second button in the row of buttons just under "SilverFast AI".

\section{Click Scan.}

The Save as... window opens. Make certain that the folder to hold the scanned images appears in the Save in: drop down box. If necessary, create a new folder by first selecting the parent folder in the Save in: box, and then clicking the Create New Folder button to the right of the Save in: box. Confirm that the correct file name or number is specified and that "TIFF ( ${ }^{*}$.tif) is selected as the file type.

NOTE: Unless a smaller file size is really helpful, TIFF is a preferable format to JPEG because information is lost when JPEG files are copied.

Click Save. The scanner will scan the area within the scan frame in high resolution and save the file to the hard drive in the designated folder.

Move the scan frame to the next film, and click on Image Autoadjust. If you are scanning a homogeneous series of negatives, you need not change the frame's size or shape. Increment the sequence number or name in the file Name as appropriate and click $\underline{S c a n}$. Confirm the folder and new file name and click Save. Continue until all of the images are scanned. If you get confused as to what the sequence number should be for any scan, click the Scan button. This will bring up the Save as... window where you can check the proper numbering of the previous scans. Then cancel the scan from this window.

\section{Close SilverFast.}

\section{Processing the Scanned Images}

It is not within the scope of this article to describe image processing in detail. However for completeness, following are very basic steps that have proven helpful in improving transmission E.M. images that come off the scanner.

The images, as they first come off the scanner, are often dull with little separation among the levels of gray and no blacks or whites. They can be improved somewhat through the use of brightness and contrast controls in PhotoShop. However brightness and contrast adjustments affect every pixel throughout the image equally and information is lost. A superior method is available through the Levels function in PhotoShop.

Open PhotoShop. Load the first image in the scanned series. Hit Ctrl-O, navigate to the first image and hit Enter.

Levels (Ctrl-L) will produce a histogram showing the distribution of gray levels in the image. Along the $\mathrm{x}$-axis are 256 channels representing 256 levels of gray from black to white. The vertical bars represent the number of pixels within each of the gray levels. The normal human eye and brain are capable of separating and distinguishing slightly fewer than 256 levels of gray.

There are three sliders under the levels $\mathrm{x}$-axis. The two outer ones specify which channel or gray level is assigned the value of pure black (left) in the output and which channel is assigned the level of pure white (right) in the output.

By moving (drag and drop) the left slider to the left most significant vertical bar, we assign the value of pure black to that darkest level of gray in the unprocessed image. Likewise by moving the right hand slider to the right most significant vertical bar, we assign the value of pure white to the brightest level of gray in the image. Automatically the computer distributes the intermediate levels of gray among the remaining 254 output channels as appropriate for that image, to make the best use of the available gray levels that the eye can distinguish. The result is an output image with blacks and whites as well as a full spectrum of intermediate gray levels. That automatic distribution of mid tones can be skewed left or right to darken or lighten the overall image by moving the middle ("gamma") slider. Contrast and brightness are improved and no information is lost.

\section{Labeling the images}

There may be identifying notation (neg. number, mag. value, etc.) on the negative. It is wise to include this portion of the negative in the scan area. However you can also add text to the scanned image.

In PhotoShop's tool menu. Select the "Horizontal type tool", which looks like a large " $T$ ". Select the following settings for the type tool:

Font

Style

$\rightarrow$ Arial, or as appropriate

Size

$\rightarrow$ Regular

Anti aliasing $\rightarrow$ None

Alignment $\rightarrow$ Left align text

Text color $\rightarrow$ Any that contrasts well with the background

Establish the text insertion point where you want the text to begin by bringing the text icon into position and clicking once. Then type in the desired text. If you are scanning a series of micrographs, you might select the text just entered and copy it to the clipboard. Then just paste it to subsequent images, incrementing any sequence numbers.

Once the images are processed they are gathered into a zipped or compressed file using Winzip, Stuffit, or the compressed folder function of Windows XP (In Windows Explorer: File | New / Compressed (zipped) Folder). We include a text file holding information (case number, patient number, etc.) pertinent to the image series. This compressed file is then transmitted by FTP to the physician requesting the service. That person can view the images on his or her computer, print them out, or include them in reports, etc. A second compressed file identical to the first is transferred to an archival quality $\mathrm{CD} / \mathrm{ROM}$ and filed in a secure location.

We have found that the above procedures have saved us considerable time, cost and filing space. But also the need to chase down the prints for required quality assurance purposes after the case is signed out has been eliminated. Our files are always complete. 

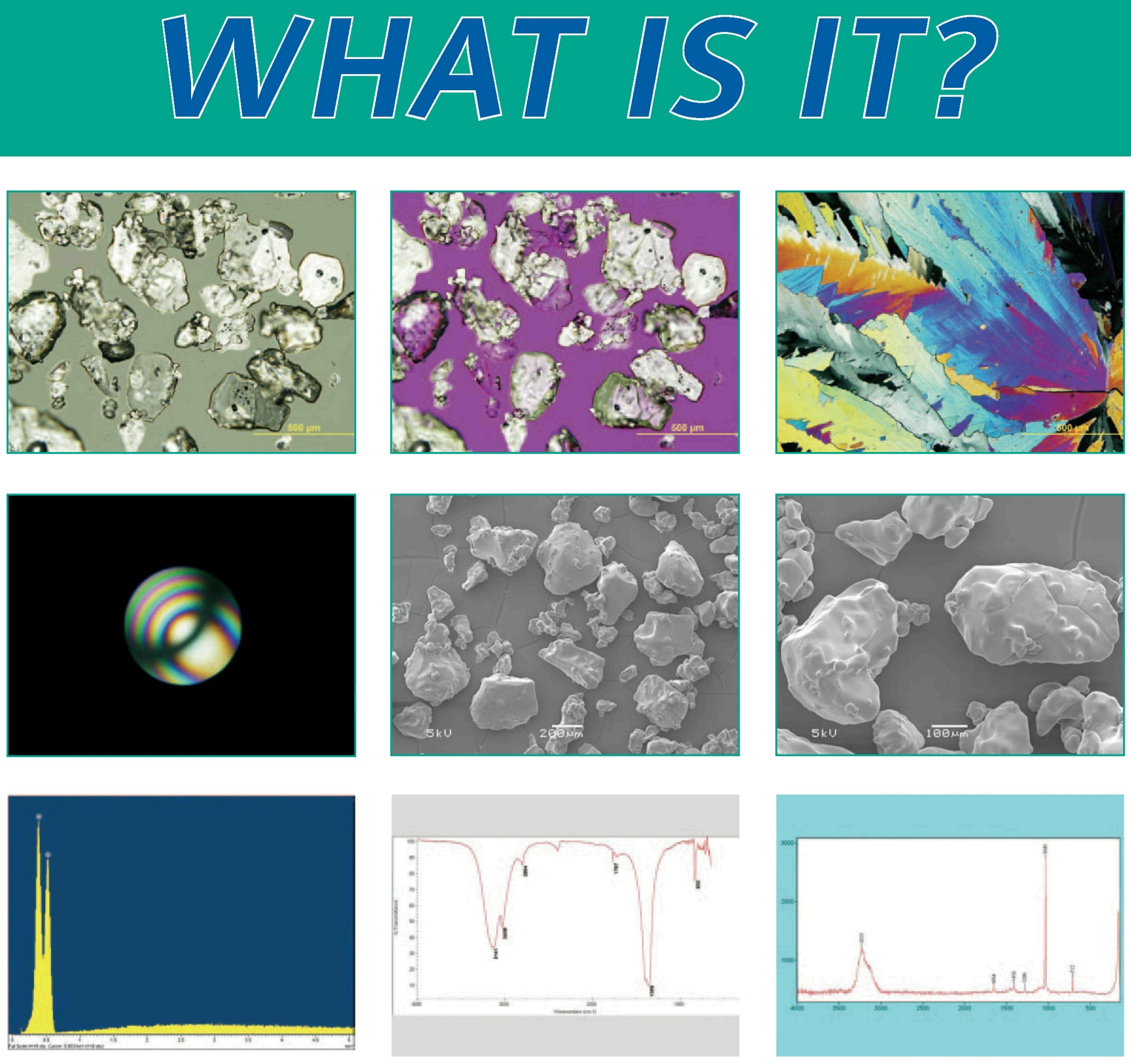

\section{FIND OUT \\ Apríl 1, 2005 \\ www.mccroneatlas.com}

McCrone Atlas of Microscopic Particles brought to you by

on MCCRONE: 

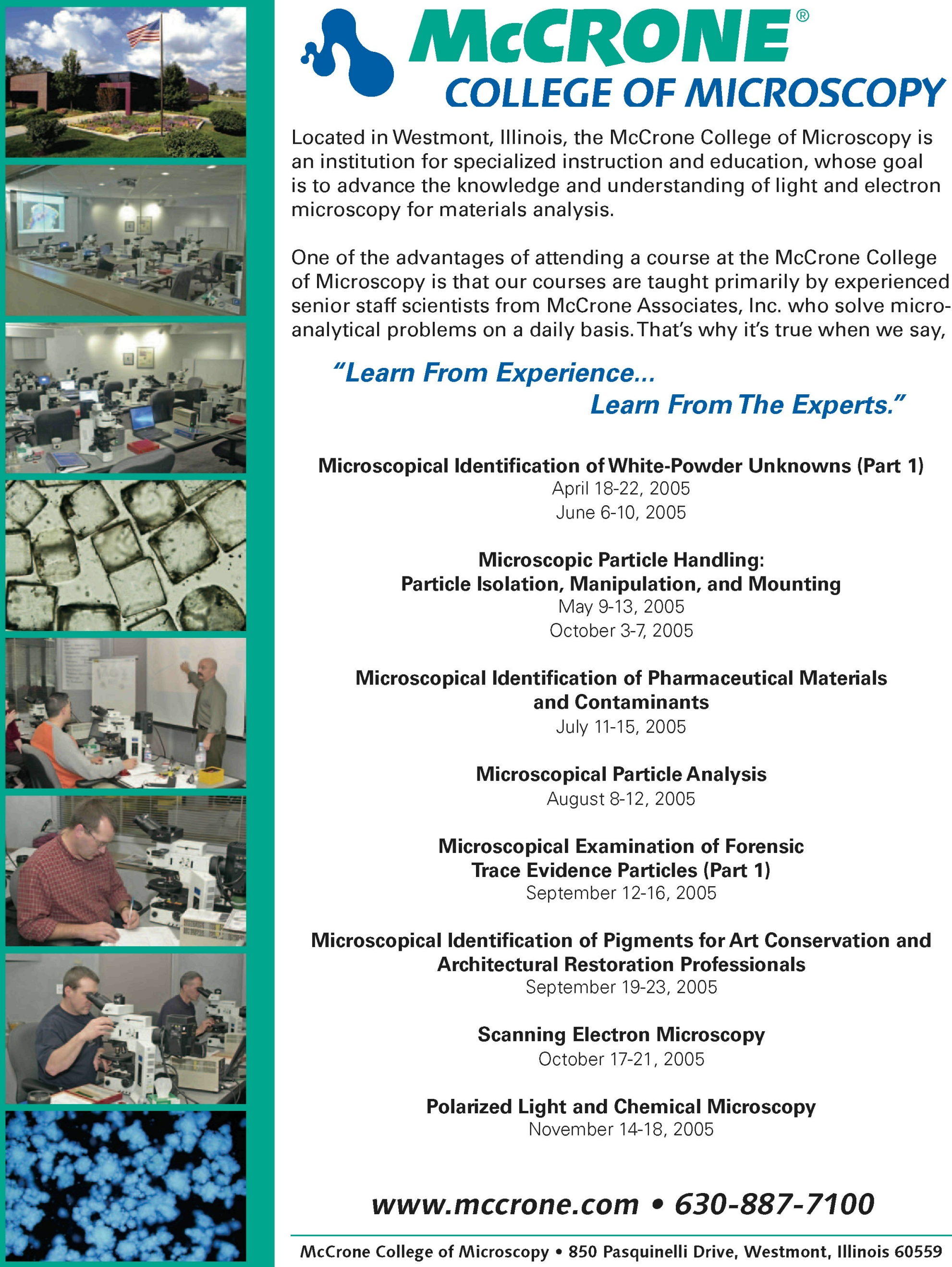

Located in Westmont, Illinois, the McCrone College of Microscopy is an institution for specialized instruction and education, whose goal is to advance the knowledge and understanding of light and electron microscopy for materials analysis.

One of the advantages of attending a course at the McCrone College of Microscopy is that our courses are taught primarily by experienced senior staff scientists from McCrone Associates, Inc. who solve microanalytical problems on a daily basis. That's why it's true when we say,

\section{"Learn From Experience... \\ Learn From The Experts."}

Microscopical Identification of White-Powder Unknowns (Part 1) April 18-22, 2005 June 6-10, 2005

Microscopic Particle Handling:

Particle Isolation, Manipulation, and Mounting

May 9-13, 2005

October 3-7, 2005

Microscopical Identification of Pharmaceutical Materials and Contaminants

July 11-15, 2005

Microscopical Particle Analysis

August 8-12, 2005

Microscopical Examination of Forensic

Trace Evidence Particles (Part 1)

September 12-16, 2005

Microscopical Identification of Pigments for Art Conservation and Architectural Restoration Professionals

September 19-23, 2005

Scanning Electron Microscopy

October 17-21, 2005

Polarized Light and Chemical Microscopy

November 14-18, 2005

wWw.mccrone.com • 630-887-7100 